\title{
Lymphopoïèse et gène E2A: le rôle spécifique d'un facteur ubiquitaire
}

Les protéines E12 et E47 sont toutes deux dérivées du gène $E 2 A$, engendrées par épissage alternatif. Il s'agit de facteurs de la famille basique hélice-boucle-hélice (b-HLH, basic helixloop-helix) à laquelle appartiennent également les différenciateurs myogéniques de la famille MyoD1. Il existe un site de fixation pour un dimère de protéines E12/E47 dans le enhancer des gènes d'immunoglobulines et, sous forme d'hétérodimères avec d'autres protéines b-HLH, E12/E47 se fixent à des motifs CANNTG* présents dans les régions régulatrices de nombreux gènes, notamment exprimés dans le muscle. Deux équipes viennent d'obtenir des souris dépourvues des produits du gène $E 2 A$ à la suite de l'invalidation homozygote du gène par recombinaison homologue (Zhuang et al., Seattle, WA, USA et Bain et al., La Jolla, CA, USA ; Baltimore, MD, USA ; Amsterdam, Pays-Bas) [1, 2]. Les résultats des deux groupes sont tout à fait convergents: les animaux naissent hypotrophiques et meurent rapidement, dépourvus de malformations notables. En fait, la principale anomalie est l'absence totale de lignée lymphocytaire B mûre, les précurseurs de cette lignée étant bloqués au stade des cellules proB CD34+, qui sont ellesmêmes diminuées environ de moitié. Au niveau de ces cellules proB, il n'y a pas de réarrangement des segments du gène des chaînes lourdes, et les

* Bô̂tes $E$ où $N$ représente $n$ 'importe quel nucléotide $C, A, G$, ou $T$. transcrits pax 5, CD19 et RAG1 sont réduits ou absents. Le gène de chaîne lourde non réarrangé engendre normalement deux types de transcrits, dont l'un débute dans le enhancer situé dans l'intron précédant les exons codant pour les parties constantes (I $\mu)$, l'autre en amont des exons codant pour les fragments de jonction $\mathrm{J}(\mu \mathrm{o})$. Les premiers types de transcrits sont en quantité réduite, les seconds en quantité normale, ce qui indique que la fixation de E121/E47 au enhancer serait indispensable à la stimulation de la transcription de type $\mathrm{I} \mu$, mais non de type $\mu \mathrm{o}$. Les transcrits $\lambda 5$, codant pour un remplaçant temporaire de chaîne légère dans les précurseurs ayant déjà réarrangé le gène de chaîne lourde mais non les gènes de chaîne légère, sont également absents. Les mécanismes du blocage peuvent être multiples: absence de réarrangement des gènes d'immunoglobulines, directement en rapport avec l'absence de fixation de E12/E47, ou secondaire au déficit en recombinase RAG 1 ; absence de Pax5, intervenant dans la non-expression de $\lambda 5$ et de la molécule d'activation CD 19, etc. Le rôle de Pax5, aussi dénommé BSAP ( $B$ lineage-specific activator protein) est attesté par le phénotype des souris pax5-/-, également obtenues par recombinaison homologue, qui est caractérisé par un blocage de la maturation lymphocytaire $\mathrm{B}$ associé à des anomalies du développement du cerveau moyen (Urbanek et al., Vienne, Autriche) [3]. En revanche, il n'existe aucune anomalie visible du systè- me nerveux central et des muscles chez des animaux E2A-/-. Ce dernier point est particulièrement important puisque l'on sait que les facteurs myogéniques de la famille MyoD1 ainsi que les facteurs impliqués dans la neurogenèse et équivalents des gènes achaete-scute de la drosophile, fondamentaux pour la neurogenèse, agissent sous la forme d'hétérodimères avec les protéines E12/E47. Le phénotype des souris E2A-/- indique que d'autres formes de dimères peuvent remplacer les hétérodimères MyoD1-E12/E47 ou achaete-scute-E12/ E47. Aucun renseignement n'est donné sur la nature de ces hétérodimères. Une autre observation surprenante est que le désordre est limité aux cellules $\mathrm{B}$ alors que le gène $E 2 A$ est actif dans toutes les cellules. Il semble bien que la forme active dans les cellules lymphocytaires B soit l'homodimère entre les produits de $E 2 A$. Il pourrait se faire que cette homodimérisation fût spécifique de la différenciation lymphocytaire $\mathrm{B}$, soit à cause d'une plus forte expression du gène $E 2 A$ dans ce lignage, soit parce que l'homodimérisation est facilitée par une protéine spécifique de la lignée $B$. De fait, la présence spécifique d'un dimère de protéine $\mathrm{E} 47$ dans les cellules $B$ a été rapportée [4]. La dissociation de cet homodimère peut être obtenue par l'intermédiaire d'un excès de protéine Id, une protéine HLH dépourvue du domaine basique de liaison à l'ADN, capable de se dimériser mais produisant ainsi des dimères sans affinité pour l'ADN ; la diminution de la syn- 
thèse de Id est d'ailleurs corrélée à la progression de la différenciation lymphocytaire B. Un chercheur américain, Sun (New York), vient de démontrer le rôle de Id... et de confirmer celui de E2A en produisant des souris transgéniques synthétisant de façon constitutive de fortes quantités de Id dans les cellules proB et $\mathrm{B}$ : le phénotype de ces animaux est pratiquement le même que celui des souris E2A-/- [5]. Quelles que soient les explications correctes des phénotypes observés chez ces animaux $\mathrm{Id}^{+} /^{+}$ou $E 2 A-/$, ces résultats soulignent deux phénomènes importants en biologie: la redondance fonctionnelle (puisque la fonction de E2A comme partenaire d'hétérodimérisation de nombreux facteurs bHLH est remplacée, chez les souris déficientes, par autre chose) et la spécificité possible de l'action de protéines ubiquitaires.

A.K.

1. Zhuang Y, Soriano, P, Weintraub $\mathrm{H}$. The helixloop-helix gene $E 2 A$ is required for $\mathrm{B}$ cell formation. Cell $1994 ; 79$ : 875-84.

2. Bain G, Robanus Maandag EC, Izon DJ, Amsen D, Kruisbeek AM, Weintraub BC, Krop I, Schlissel MS, Feeney, AJ, van Roon M, van der Valk M, te Riele HPJ, Berns A, Murre C. E2A proteins are required for proper B cell development and initiation of immunoglobulin gene rearrangements. Cell $1994 ; 79$ : 885-92.

3. Urbanek P, Wang ZQ, Fetka I, Wagner EF, Busslinger M. Complete block of early B cell differentiation and altered patterning of the posterior midbrain in mice lacking Pax5/BSAP. Cell $1994 ; 79: 901-12$.

4. Murre C, Voronovan A, Baltimore D. B-cell and myocyte-specific E2-box-binding factors contain E12/E47 like subunits. Mol Cell Biol 1991; 11: $1156-60$.

5. Sun XH. Constitutive expression of the IdI gene impairs mouse B cell development. Cell $1994 ; 79: 893-900$.

$m / s n^{\circ} 3$, vol. 11, mars 95

\section{BRÈVES}

प Une base cellulaire à la protection exercée par certaines hémoglobinopathies vis-à-vis du paludisme. Le caractère protecteur des hémoglobinopathies à l'état hétérozygote vis-à-vis du paludisme a été évoqué de longue date par Allison sur la base d'enquêtes épidémiologiques et a donné lieu au concept de polymorphisme équilibré. Bien que généralement admise, cette protection a pu être contestée par certains auteurs arguant que l'infestation parasitaire est aussi fréquente chez les drépanocytaires hétérozygotes (AS) que chez les sujets témoins, et parfois de densité identique. Plus récemment, on a cependant pu montrer que la protection s'exerce en fait sur le jeune enfant AS avant qu'il n'ait établi son immunité, et qu'elle réduit la mortalité due à la forme grave qu'est le paludisme cérébral [1]. Une base moléculaire est proposée à ce caractère protecteur : dans la circulation profonde, quand la pression d'oxygène est basse, les érythrocytes AS inhibent la croissance du parasite, entraînant mort cellulaire et séquestration splénique du globule rouge parasité. En ce qui concerne les thalassémies, on n'a formulé que des hypothèses. Les études de ces dernières années avaient montré le rôle majeur, au cours du paludisme cérébral, d'une formation spontanée de rosettes autour des érythrocytes infestés, ainsi que l'existence d'anticorps anti-rosette dans les formes atténuées de paludisme. Pouvait-on montrer, au cours des maladies "protectrices", une modification de ce phénomène? Cela $a$ été étudié sur une gamme de malades, drépanocytaires SS, AS et SC, thalassémiques $\alpha$ et $\beta$, mais aussi témoins AA microcytaires par carence en fer. On a ainsi mesuré la proportion de rosettes formées, leur taille (nombre d'érythrocytes agglutinés) et leur résistance à la dissociation ou propension à se reformer après dissociation [2]. Les résultats semblent sans ambiguïté chez tous les thalassémiques, chez qui les rosettes sont à la fois moins nombreuses et moins cohérentes. Un phénomène similaire est retrouvé chez des sujets microcytaires non thalassémiques et pourrait expliquer des cas de résistance au paludisme observés au cours de malnutrition. Chez tous les sujets porteurs d'hémoglobine $S$, un phénomène analogue est retrouvé après un cycle de désoxygénation et réoxygénation simulant la physiopathologie et pourrait être rapportée à des modifications même mineures des propriétés mécaniques du globule rouge. Les mécanismes en cause ne peuvent que faire l'objet d'hypothèses. La formation de rosettes est due à l'interaction entre protéines de l'érythrocyte infesté et récepteurs des cellules agglutinées. Y a-t-il, sur les globules rouges microcytaires, moindre accessibilité ou moindre expression de ces récepteurs? Dans le cas des cellules SS ou AS, c'est sûrement leur accessibilité qui se trouve modifiée par la distorsion et la rigidification cellulaires. Au mécanisme moléculaire mis en cause dans le cas de la drépanocytose s'ajoute donc un mécanisme cellulaire qui semble jouer dans tous les cas de microcytose et de rigidité mécanique. Restent, non encore expliquées, les protections observées au cours d'enzymopathies, de déficit en glucose 6-phosphate déshydrogénase, ou dans le cas d'anomalies des protéines membranaires telles que l'ovalocytose du Sud-Est asiatique.

[1. Grau G, et al. médecine/sciences 1990 ; 6 (suppl 7) : 52-8.]

[2. Carlson J, et al. Blood 1994 ; 84 : 3909-14.] 